\title{
AUTHOR INDEX (boldface number indicates first author)
}

Alderliesten, C., 215

Alekseev, A. Yu., 767

Alexandrovskiy, A. L., 583, 991

Ambers, J., 591

An, Z., 905

Anderson, R., 193

Anton, G., 231

Aravena, R., 1013

Arslanov, Kh. A., 177, 289

Barbetti, M., 351

Bartosiewicz, L. 659

Bazarova, V. B., 775, 841

Beavan, N. R., 601

Beer, J., 849

Bendat, M., 615

Benner, B. A., Jr., 381

Benz, M., 231

Berger, R., 615

Biegalski, S. R., 3, 265, 381

Bitterling, M., 239

Blasche, S., 231

Boaretto, E., 915

Bokovenko, N. A., 713

Bonani, G., 803, 849

Borziak, I., 649

Boschin, M. T., 35

Boulet, R., 1013

Brauer, A., 803

Bronk Ramsey, C., 59, 35, 283, 461

Bruins, H. J., 621

Bull, D., 781

Burky, R. R., 11

Buzinny, M., 373

Cahoon, D. R., 381

Carmi, I., 77, 707, 945

Caulfield, S., 629

Chamyal, L. S., 819

Chichagova, O. A., 583, 991

Chugunov, K. V., 713

Conny, J. M., 381

Cook, G. T., 21, 193, 289, 331, 447

Cooper, M. C., 781

Currie, L. A., 3, 113, 265, 381

Dahm, J., 975

Damblon, F., 649

Damon, P. E., 343

Dams, R., 103

de Jong, A., 215

de Leeuw, J. W., 985

Dergachev, V. A., 571, 759, 767

Dibb, J. E., 381

Donahue, D. J., 905

Doubrava, M. R., 561

Dreyer, G., 641

Druffel, E. R. M., 29
Dugmore, A. J., 21

Eastoc, C. J., 343

Eglinton, T. I., 95

Eijgenhuijsen, E. M., 113

Eitel, B., 795

Elder, K. L., 223

Enerson, T. B., 167

Erlandsson, B., 433

Erlenkeuser, H., 517

Erler, G., 231

Etchegoyen, A., 44

Facorellis, Y., 963

Fernandez Niello, J. O., 35

Finckh, E., 231

Fischer, L., 231

Fletcher, R. A., 3

Fokker, W., 283

Furukawa, M., 701

Futo, I., 659

Gagnon, A. R., 223

Gal, Z., 707

Ganzey, L. A., 775, 841

Geirsdóttir, Á., 865

Geyh, M. A., 475, 795, 921

Golser, R., 255, 265, 273

Gomes, B. M., 1013

Gorczyca, Z., 129

Goslar, T., 551

Gouveia, S. E. M., 1013

Grebennikova, T. A., 775, 841

Griffin, S., 29

Grootes, P. M., 85, 239, 517

Grosjean, M., 921

Gröning, M., 295

Gulliksen, S., 289

Görsdorf, J., 641, 713

Haas, H., 167, 561

Haesaerts, P., 649

Hajdas, I., 803

Hare, P. E., 11

Harkness, D. D., 201, 289, 331, 781, 873

Hartung, U., 641

Harđardöttir, J., 865

Hasselberg, P., 239

Hassink, J., 1023

Hayes, J. M., 95

Head, M. J., 45, 905

Hedges, R. E. M., 35, 51, 59, 283

Heinemeier, J., 865, 915

Hellborg, R., 433

Hendrix, V., 735

Hertelendi, E., 439, 659, 855

Higham, T. F. G., 45, 975

Hille, P., 273

Hiller, A., 721 
Hogg, A. G., 45, 975

Hoper, S. T., 45

Hormes, A., 809

Horvatincic, N., 399

Horváth, F., 659

Hsu, N. C., 381

Hughen, K. A., 483

Hughes, M. K., 343

Ikeda, A., 933

Jelen, K., 129

Jiang, M., 849

Jones, R. I., 781

Jull, A. J. T., 675, 905

Jöris, $0 ., 495$

Kalin, R. M., 343

Kankainen, T., 289

Kashgarian, M., 483

Kemp, A. E. S., 781

Kerscher, H. 231

Kilian, M. R., 535

Kirner, D. L., 11

Kitagawa, H., 505

Klaver, E. R., 535

Klein, M. 231

Klinedinst, D. B., 265, 381

Klochko, V. I., 667

Klouda, G. A., 3, 113, 265, 381

Kobal, I., 439

Kobayashi, T., 825

Kocharov, G., 177

Koff, T., 833

Kojima, S., 933

Koopmans, R., 283

Korotky, A. M., 775, 841

Kotva, A., 231

Kouwenberg, J. H. M., 535

Kovaliukh, N. N., 211, 391, 583, 667

Krajcar-Bronić, I., 399

Kramer, S. J., 299, 999

Krenke, N., 583

Kretschmer, W., 231, 933

Kristensen, P., 865

Kromer, B., 351, 963

Kronfeld, J., 945

Kuc, T., 129, 417

Kusumgar, S., 819

Kutschera, W., 255, 265, 273

Kuzmin, Y. V., 313, 675

Küster, H., 231

Lagiglia, H. A., 693

Laj, C., 517

Lange, M., 687

Lawson, E. M., 781

Le Clercq, M., 295

Lebedeva, L. M., 571, 713

Lehman, S. J., 483

Leigart, M., 231
Likhtarev, I., 373

Liu, T., 849

Llamazares, A. M., 35

Long, A., iil(1), 57, 343, 693

Losí, I., 373

Lowe, J. J., 873

Mackenzie, A. B., 447

Maniatis, Y., 963

Marsadolov, L. S., 571

Martin, P. S., 693

Masuda, K., 177

Matthews, N. M., 781

Mattsson, S., 433

Mazaud, A., 517

McCartney, M., 425

McCormac, F. G., 45

McDonald, P., 447

McNamara, N., 425

McNichol, A. P., 61, 95, 223, 247

Meadows, P. S., 447

Megens, L., 985

Michczynski, A., 137

Miller, B. F., 201

Milton, G. M., 299, 999

Milton, J. C. D., 299

Mintz, E., 77

Mitchell, P. I., 629

Mizutani, Y., 933

Moens, L., 103

Mokhova, L. M., 775, 841

Mook, W., iil(2)

Morgenroth, G., 231

Morávek, J., 439

Motzenbecker, I., 667

Mous, D. J. W., 283

Muraki, Y., 177

Murata, T., 177

Mądry, W., 551

Nadeau, M.-J., 85, 239, 517

Nagler, A., 713

Nakamura, T., 701, 825, 933

Naruse, Y., 177

Naysmith, P., 447

Neary, M. P., 183

Negendank, J., 803

Nielsen-Marsh, C., 35

Nishiyama, T., 177

Noakes, J. E., 183

Obelic, B., 399

Oda, H., 701, 933

Ohta, T., 933

Okuno, M., 825, 933

Olsson, I. U., 143

Omoto, K., 151

Orlova, L. A., 313, 675

Overpeck, J. T., 483

O'Donnell, R. G., 629 
O'Sullivan, P. E., 781

Parker, A., 615

Parzinger, H., 713

Passo, C. J., 193

Pawlyta, J., 201

Pazdur, A., 137, 201

Pearson, A., 61, 247

Peglar, S. M., 781

Peristykh, A. N., 343

Persson, P., 433

Pessenda, L. C. R., 1013

Peterson, L. C., 483

Pettitt, P. B., 35

Possnert, G., 767

Priller, A., 255, 265, 273

Puchegger, S., 273

Punning, J.-M., 833

Raczky, P., 659

Raj, R., 819

Rakowski, A. Z., 201

Razjigaeva, N. G., 775, 841

Remmele, S., 351

Renssen, H., 535

Reynaud-Farrera, I., 535

Ribeiro, A. S., 1013

Rieck, A., 239

Roberts, D., 193

Rogojin, V., 945

Rom, W., 255, 265, 273

Rud, N., 865

Römkens, P. F. A. M., 1023

Sarnthein, M., 517

Schleicher, M., 85, 239, 517

Schlüchter, C., 475, 809

Schneider, R. J., 61, 95, 247

Schoon, A., 85

Schotterer, U., 921

Scott, E. M., 289, 331, 425

Segal, D., 77, 707

Sementsov, A. A., 571, 713, 759, 767

Shalem, D., 707

Shen, C., 849

Shine, A. J., 781

Shore, J. S., 21

Skog, G., 433

Skripkin, V. V., 211, 391, 667

Slavik, O., 439

Smithline, H., 707

Southon, J. R., 11, 483

Sparks, R. J., 601

Spaulding, J. D., 183

Spurk, M., 351

Steier, P., 255, 265, 273

Stenström, K., 433

Stocker, T. F., 359, 809

Stäuble, H., 721

Sulerzhitsky, L. D., 583, 675, 775, 841
Sun, Y., 849

Sveinbjörnsdóttir, Á. E., 865, 915

Svingor, E., 659

Szabo, J., 77

Sümegi, P., 855

Takenaka, C., 367

Talerko, N., 373

Taylor, R. E., 11

Theodorsson, P., 157

Thorling, L., 915

Thornberg, C., 433

Timofeev, V. I., 759

Titus, R. L., 167

Toniello, V., 351

Tsigankov, N., 373

Turney, C. S. M., 873

Uchrin, G., 439

Van den Broek, R., 283

Van der Borg, K., 215

Van der Plicht, J., vil(1), 103, 295, 391, 505, 535, 571 ,

$583,621,649,985,1023$

Van Geel, B., 535

Van Klinken, G. J., 35, 51, 59

Van Strydonck, M. J. Y., 735

Vandeputte, K., 103

Vartanyan, S., 289

Vasiliev, S. S., 571

Vasil'chuk, A. C., 883, 895

Vasil'chuk, Y. K., 883, 895

Voelker, A. H. L., 517

Vokal, B., 439

Volent, G., 439

Von Reden, K. F., 61, 95, 247

Vycudilik, W., 273

Waldren, W. H., 735

Waterbolk, H. T., 535

Webb, R. E., 749

Weissenbök, R., 3, 265

Weninger, B., 495

Wild, E., 255, 265, 273

Wills, J. S. C., 95

Wilson, A. T., 953

Wolstenholme, A., 447

Wright, D. G., 359

Yadav, M. G., 819

Yechieli, Y., 915

Yi, W., 849

Yokota, K., 933

Yonenobu, H., 367

Zaitseva, G. I., 289, 571, 713, 759, 767

Zarrabi, K., 167

Zheng, Y., 61

Zhou, W., 905

Zimnoch, M., 417

Zolitschka, B., 803

Zolnikov, I. D., 313 